\title{
La formación de podosomas y la adhesión de leucocitos es mediada por la Quinasa Ligada a Integrinas en la enfermedad renal crónica $(\mathrm{ERC})$.
}

Sofía Campillo1, 2, a , Lourdes Bohorquez 1, 2, b , Elena Gutiérrez-Calabrés 1, 2, 3, c, Diego GarcíaAyuso 1, 2, d, David Vaillo1, 2, 3, e, Mercedes Griera', 2, f, Sergio de Frutos ${ }^{1,2,3,9}$, Manuel RodríguezPuyol $^{1,2, \text { h, Diego Rodríguez-Puyol }}{ }^{2,3,4, \text { i }, \text { Laura Calleros }}{ }^{1,2, j}$

1. Departmento de Biología de Sistemas, Unidad de Fisiología, Universidad de Alcalá de Henares, Alcalá de Henares, Madrid, España. 2. Instituto Ramón y Cajal de Investigaciones Sanitarias (IRYCIS), Fundación Renal Iñigo Álvarez de Toledo (FRIAT), REDinREN del Instituto de Salud Carlos III, y NOVELREN de la Comunidad de Madrid, Madrid, España. 3. Fundación para la Investigación Biomédica, Hospital Universitario Príncipe de Asturias, Alcalá de Henares, Madrid, España. 4. Unidad de Nefrología del Hospital Universitario Príncipe de Asturias y Departamento de Medicina de la Universidad de Alcalá de Henares, Alcalá de Henares, Madrid, España.

a.sofia.campillo@edu.uah.es b. lourdes.bohorquez@uah.es c.elena.gutierrez@edu.uah.es

d. diegogarcia2195@gmail.com e.david.vaillo@uah.es f. mercedes.griera@uah.es g.sergio.frutos@uah.es

h.manuel.rodriguez@uah.es i. drodriguez.hupa@gmail.com j. laura.calleros@uah.es

V Congreso de Señalización Celular, SECUAH 2020.

16-18 de marzo, 2020. Universidad de Alcalá. Alcalá de Henares, Madrid. España.

Palabras clave: Enfermedad Renal Crónica; Enfermedad Cardiovascular; Quinasa Ligada a Integrinas (ILK); podosomas

\section{Resumen}

Los pacientes con ERC tienen un mayor riesgo de desarrollar enfermedades cardiovasculares. Las toxinas urémicas unidas a proteínas, como el $p$-cresol (pc) y el indoxil sulfato (IS), no se pueden eliminar mediante los métodos de hemodiálisis convencional y producen disfunción endotelial induciendo la adhesión y extravasación de leucocitos al endotelio. Dichos procesos pueden llevarse a cabo mediante estructuras de adhesión dinámicas denominadas podosomas. La quinasa ligada a la integrina (ILK) vincula los receptores de adhesión celular, las integrinas y los factores de crecimiento con el citoesqueleto de actina y con diferentes vías de señalización. El objetivo de este estudio fue investigar la implicación de ILK en la formación de podosomas y la adhesión de leucocitos en condiciones urémicas que simulan la ERC. Los experimentos in vitro se realizaron en la línea celular humana de monocitos, THP-1. Las células fueron incubadas con IS $\left(25\right.$ y $\left.100 \mu \mathrm{g} \mathrm{ml}^{-1}\right)$ y pc $\left(10\right.$ y $\left.100 \mathrm{~g} \mathrm{ml}^{-1}\right)$ en diferentes tiempos. Se determinaron la viabilidad celular, los niveles de expresión de ILK por Western Blot y RT-qPCR y su actividad quinasa, mediante niveles de fosforilación de P-GSK-3 $\beta$ (Ser9) por Western Blot. Además, ILK fue delecionada con un siRNA específico para analizar la formación de podosomas y la adhesión celular a una matriz de fibronectina, la transmigración de los monocitos y la degradación de la matriz extracelular. También se estudió la colocalización de ILK con cortactina, proteína específica de podosomas. Observamos que las toxinas urémicas no inducen toxicidad en las células THP-1. La actividad de ILK aumentó con el tratamiento de toxinas urémicas a bajas y altas concentraciones, sin cambios en su expresión proteica. La adhesión de los monocitos, la formación de podosomas, la transmigración celular y la degradación de la matriz extracelular fueron significativamente mayores con el tratamiento con toxinas a dosis bajas y altas, mientras que este aumento no se observó con la deleción de ILK. La colocalización de ILK con cortactina confirmó la implicación de ILK en la estructura de los podosomas. Para los experimentos ex vivo, se inyectó tamoxifeno (ratones cKD-ILK) o vehículo (ratones WT) a ratones CRE-LOX, para conseguir la deleción de ILK. Se aislaron las células mononucleares de sangre periférica (CMSP) de los ratones, se trataron con toxinas urémicas y se analizó la adhesión celular a una matriz de fibronectina. En las CMSP de los ratones cKD-ILK se observó una menor adhesión celular, en comparación con los ratones WT, y las toxinas urémicas indujeron un aumento significativo de la adhesión de las CMSP, que fue significativamente menor en las CMSP de los ratones CKD-ILK. Estos datos sugieren que ILK desempeña un papel crítico en los procesos requeridos para la extravasación de leucocitos inducido por la acumulación de las toxinas urémicas y que podría ser una diana terapéutica potencial para el tratamiento del daño vascular asociado con la ERC.

Cita: Campillo, Sofía; Bohorquez, Lourdes; Gutiérrez-Calabrés, Elena; García- Ayuso, Diego; Vaillo, David; Griera, Mercedes; de Frutos, Sergio; Rodríguez-Puyol, Manuel; Rodríguez-Puyol, Diego; Calleros, Laura (2020) La formación de podosomas y la adhesión de leucocitos es mediada por la Quinasa Ligada a Integrinas en la enfermedad renal crónica (ERC). Actas del V Congreso de Señalización Celular, SECUAH 2020. 16-18 de marzo, 2020. Universidad de Alcalá. Alcalá de Henares, Madrid. España. dianas 9 (1): e202003b08. ISSN 1886-8746 (electronic) journal.dianas.e202003b08 http://www3.uah.es/dianas?e202003b08.

URI http://hdl.handle.net/10017/15181. DOI https://doi.org/10.37536/DIANAS.2020.9.1.74

Copyright: @ Campillo S, Bohorquez L, Gutiérrez-Calabrés E, García--Ayuso D, Vaillo D, Griera M, deFrutos S, Rodríguez-Puyol M, Rodríguez-Puyol D, Calleros L. Algunos derechos reservados. Este es un artículo open-access distribuido bajo los términos de una licencia de Creative Commons Reconocimiento-NoComercialSinObraDerivada 4.0 Internacional. http://creativecommons.org/licenses/by-nc-nd/4.0/ 